\title{
Process for the Continuous Production of Fatty Acid Esters via Reactive Distillation
}

\author{
Claudia von Scala*, Peter Moritz, and Peter Fässler \\ Sandmeyer Prize Laureates 2003
}

\begin{abstract}
Sulzer Chemtech develops chemical processes in close collaboration with its customers that lead to lower operating and investment costs as well as reduced environmental impact, while at the same time increasing the yield. In this context, innovative technologies and collaboration in global teams play an important role. This is exemplified by the continuous production of fatty acid esters using reactive distillation.
\end{abstract}

Keywords: Esterification · Fatty acid esters · Katapak · Reactive distillation - Sandmeyer Prize

\section{Fatty Acid Esters}

Food, textiles, and cosmetic applications comprise the largest use of fatty acid esters. Other uses for fatty acid esters are in the processing of rubbers and plastics, metal treatment, synthetic lubricants, and various household products. Methyl esters are used mainly as intermediate raw material for further chemical conversion into other esters, amides, ester sulfonates, and fatty alcohols.

Fatty acids, mainly from vegetable sources such as palm trees, soybean, coconuts, rapeseed or sunflowers, are mainly produced in the tropical belt, especially in South East Asia, India, China, and Brazil. Processing into fatty acid esters esterification - is done mainly in Europe, Japan, or the USA.

In the esterification reaction, a fatty acid (e.g. palmitic, myristic, stearic acid) reacts with an alcohol (e.g. 2-propanol, ethanol, butanol, 2-ethyl-1-hexanol) to form the correspondent fatty acid ester and water. This reaction is equilibrium limited, i.e. if at least one of the products is not removed, the conversion will not be complete.

${ }^{*}$ Correspondence: Dr. C. von Scala Sulzer Chemtech Ltd P.O. Box 65

$\mathrm{CH}-8404$ Winterthur

Tel: +4152262 6141

Fax: +4152 2620068

E-Mail: claudia.vonscala@sulzer.com

\section{Reactive Distillation Processes}

Reactive distillation, i.e. the combination of chemical reaction and distillative product separation in one equipment, offers several advantages over conventional processes in which the reaction and the product separation are done in series, especially for reactions limited by equilibrium constraints. As the products in reactive distillation are continuously separated from the reaction zone, no limiting chemical equilibrium can be established and thus the reaction velocity is maintained at a high rate, resulting in greater yields. Other benefits of reactive distillation can include the minimization of side reactions and the utilization of the heat of reaction for the mass transfer within the same column. Therefore the capital investment and operating costs are significantly lower with reactive distillation than for conventional processes. Examples for successful applications of reactive distillation have, among others, been reported for esterifications, etherifications, alkylations and isomerizations [1].

Sulzer Chemtech has developed a special structured catalytic packing, named Katapak, to be used in reactive distillation columns (Fig. 1). In this packing, the catalyst particles are immobilized between two sheets of metal wire gauze. Therefore, conventional catalyst particles can be used, e.g. the ones normally recommended by the process or lisense owner. Technical information of this packing type and further field of applications can be found in the article of Götze et al. [2]. Furthermore, Sulzer Chemtech has developed many processes using reactive distillation. The scope of supply for the reactive distillation technology provided by Sulzer Chemtech can include the process design, pilot tests and test runs, basic engineering, and the supply of Katapak and other distillation column internals. The design of these plants incorporates modern methods for process synthesis. In addition, Sulzer Chemtech has a broad know-how and experience in the distillation of fatty acids, gained during the last 30 years of business in this field.



Fig. 1. Katapak, structured catalytic packing

\section{Conventional Semi-Batch Process}

Esterification of fatty acids with different alcohols is nowadays mainly performed in a semi-batch mode in large stirred tank 
reactors. A large vessel is filled with the fatty acid mixture to be esterified and heated up to temperatures above $100{ }^{\circ} \mathrm{C}$. Catalyst is added to the mixture and the whole batch is held at reaction temperature. Alcohol is continuously fed to the reaction mixture and the resulting water is continuously removed from the column together with the excess alcohol by a column situated above the vessel. Through continuous removal of the water produced during the process it is possible to drive the reaction to completion. At the end the reaction mixture is further heated and vacuum is applied to remove the excess alcohol and water still present in the batch.

While batch processes have the advantage of flexibility in multi-product operation for small production capacities, their lower productivity is limiting for larger production volume. Further disadvantages of batch processes are the product quality degradation due to relatively long exposure to heat and the necessity of emptying, cleaning and refilling between each batch. The catalysts used are normally liquid acids, e.g. sulphuric acid. These acids have to be neutralized and separated, generating large amounts of waste products and wastewater.

\section{Development of the New Process}

One of the most important producers and processors of palm oil and palm oil products in Malaysia planned to increase its portfolio with fatty acid esters and approached Sulzer Chemtech Singapore in the middle of 2000. Sulzer's idea to develop a new continuous process with reactive distillation utilizing solid catalysts instead of building a conventional system readily attracted the attention of the customer. Until now, this technology had not been implemented for the production of fatty acid esters. The Malaysian customer was, however, convinced of the potential advantages of the technology, so that at the beginning of 2001 he placed an order for the process development. Under the leadership of Sulzer Chemtech, an international team of specialists from Asia and Europe started work on the new technique.

\section{Kinetic Tests}

The first task was to find a suitable solid catalyst for the esterification reaction. Other reactive distillation processes developed, e.g. for the production of ethyl and butyl acetate, use ion exchange resins which are only stable up to temperatures of about $100-120{ }^{\circ} \mathrm{C}$. The reactivity of fatty acids is, however, lower compared with shorter acid molecules, and in order to achieve sufficient conversion it was necessary to find solid acid catalysts which are active in the temperature range of 120-140 ${ }^{\circ} \mathrm{C}$. By-product formation was also an issue to be considered. A catalyst screening with various potential solid acids was performed at the University of Oldenburg, Germany, in February 2001. The catalyst chosen was a Rohm and Haas cation exchange resin especially developed for the above temperature range. The reaction kinetics were also determined and used to plan the pilot test program.

\section{Pilot Tests}

After a suitable catalyst was found, the pilot reactive distillation experiments were started in June 2001. In these experiments a part of the future production plant was reproduced in small scale to find the optimal process conditions and to determine the influence of the most important design parameters, like reflux ratio and internal column loads on the behaviour of the system. The first pilot tests were performed in a reactive distillation column with $50 \mathrm{~mm}$ diameter at atmospheric pressure. These tests showed that a higher temperature, i.e. a higher pressure, in the reactive distillation column was required. The general tendencies of the system, like the effect of reactant ratio and other distillation parameters, were determined.

Following the pilot tests at ambient pressure, an additional test series was carried out in August 2001 at a higher pressure (between 3 and 5 bars), with the aim to determine the optimal reaction temperature in the reactive distillation column. The produced ester was distilled and the resulting product was sent to the customer for quality analysis. The vapour pressure of the ester was also determined. All the data produced in these tests enabled Sulzer Chemtech to carry out the scale-up from pilot to production scale.

\section{Long-Term Activity Tests}

A long-term activity test with the chosen catalyst was also undertaken to determine its stability at operating conditions. For this, the chosen catalyst was placed in a pressure vessel. The reactants were added and the closed vessel placed in an oven to reach operating temperature and pressure. From time to time a sample was taken and sent to the catalyst manufacturer for analy- sis. About $15 \%$ of the sites were lost between the first and the second samples, but the decrease in reactivity was less intense in the next samples and stabilized with time.

\section{Final Design}

In October 2001, after the evaluation of the pilot tests and based on the process simulation results, the equipment of the plant was designed. Already by the end of 2001 it was possible to present to the customer an offer for the production plant. In March 2002, after clarification of the final details, the customer ordered the plant, which is now in the start-up phase. Sulzer Chemtech has also applied for a patent for this process.

The total development time of the process of less than two years is extremely short. The development time of similar processes, from the synthesis in laboratory scale to commercial realization, is normally significantly longer. Through the combination of experiences in the field of reaction, distillation and membrane technology a process was realized which has the potential to replace in many cases the conventional batch production of fatty acid esters.

\section{Process Description}

The following description shows the basic principles and suggests a possible process set-up. Depending on (among others) the raw material composition, the alcohol used, the site conditions, and the product specifications, various alternatives can be proposed, fitted to the customer's individual requirements.

In this example, the esterification of isopropyl palmitate with isopropyl alcohol is described (Fig. 2): Palmitic acid (PA) is first reacted with isopropyl alcohol (IPA) in a pre-reactor. The pre-reacted stream is then introduced into the reactive distillation column, which operates at slight elevated pressure. Excess IPA is added below the reactive section of the reactive distillation column to increase conversion and enhance the stripping of the formed reaction water.

At the top of the column a mixture of reaction water and IPA is obtained, together with small amounts of diisopropylether (DIPE). At the bottom of the column a mixture of isopropyl palmitate (IPP) and unreacted PA with a small amount of IPA is obtained. For this task a special tailored Katapak packing was used, in which a catalyst amount of more than 50 vol.- $\%$ in the reaction zone is reached. This allows a significant reduction of the size of the reactive dis- 


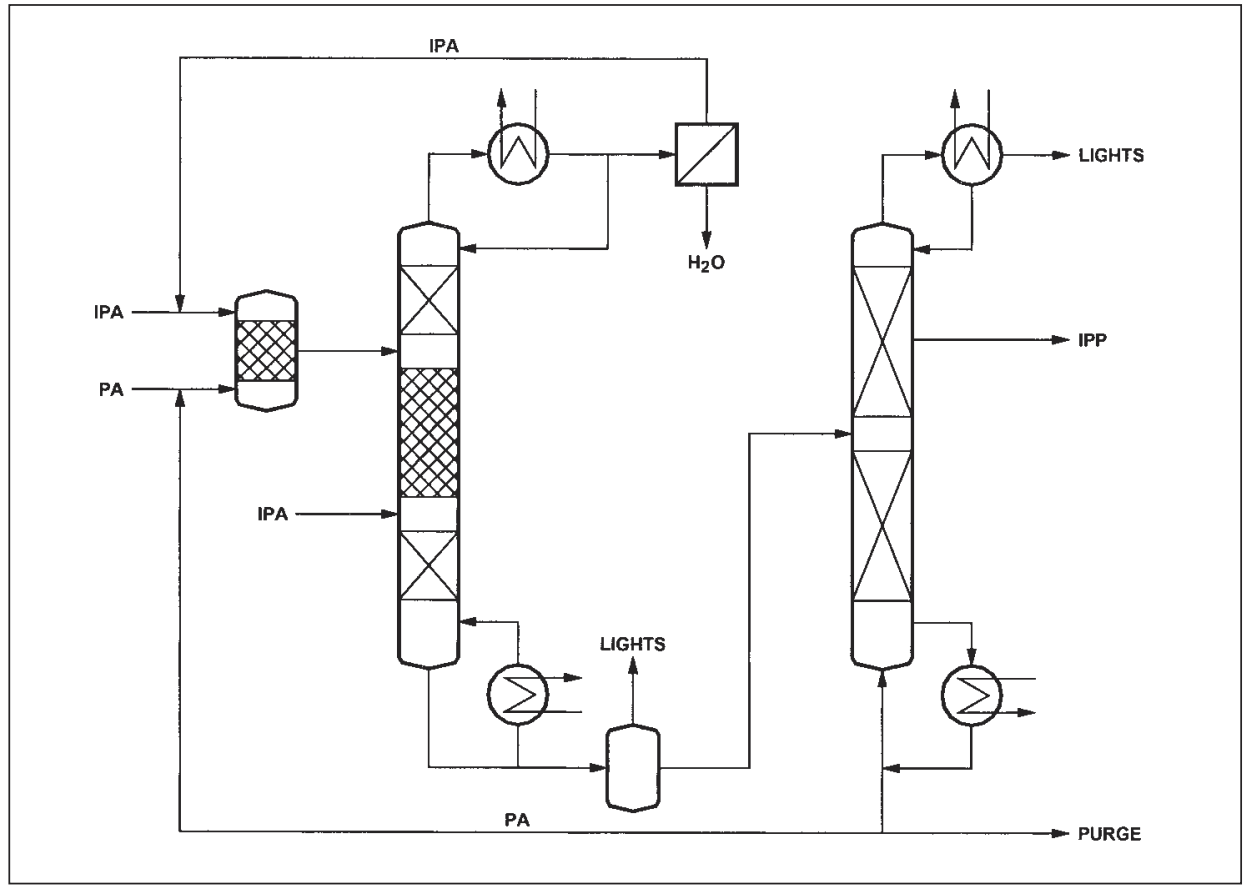

tillation column and leads to a compact construction of the plant.

The bottom product of the reactive distillation column is fed to a flash unit where the remaining IPA is separated from the mixture. This recovered IPA can be fully recycled to the process. The water/IPA mixture with traces of DIPE leaving the top of the reactive distillation column is treated in a membrane system. Water is pervaporated through the membrane and the dry IPA can be recycled to the pre-reactor or to the reactive distillation column. A purge is provided to eliminate the DIPE and other low boiling components which may be present in the feed.

IPP and PA leaving the flash unit are separated in a distillation column operated under vacuum. Special design features ensure superior IPP product quality. Unreacted PA, collected at the bottom of the distillation column, is collected and recycled to the process, whereas a heavies purge prevents the accumulation of impurities in the process.

The same esterification method can be used for any other type of purified fatty acid. A similar approach can be used with methanol or ethanol. In this case no membrane unit is needed, but an additional distillation column to separate the alcohol from the water. Other alcohols like butanol or 2-ethyl-hexanol can be separated by phase separation from the produced water.

\section{Advantages of the New Process}

The combination of reaction and separation by distillation in one reactive distillation column allows a continuous produc- tion, with reduced processing time. This leads to constant high product quality and at the same time simpler maintenance and process control, which is especially worthwhile for larger production capacities. Well defined and narrow residence time under gentle conditions throughout the whole plant minimizes degradation of the fatty acids and fatty acid esters. No neutralization, separation or recycling of catalyst is necessary. There is no necessity to empty waste streams to the absolute minimum. The energy consumption of the new process is only half of the conventional batch one. Also the size of the plant could be drastically reduced. For the treatment of the distillate stream from the top of the reactive distillation column - a mixture of isopropyl alcohol and water - a membrane module is used. The so-called pervaporation through a semi-permeable membrane can replace two distillation columns, reducing the total investment costs of the plant. It also allows a direct recycle of surplus alcohol.

\section{Summary}

Sulzer Chemtech showed with this development that, besides the broad knowhow in the field of process engineering, also innovative engineering achievements and the coordination and management of complex projects in international teams are key competencies of the company. Only through the close cooperation with the client, partners and suppliers in a very early stage, was it possible to develop a new process in extremely short time and to build it in commercial scale. The quality of the and clean the equipment, reducing the
Fig. 2. New process. The new process consists of pre-reactor, reactive distillation column, membrane module, flash unit, and vacuum distillation column.

performed work was acknowledged with the Sandmeyer Prize of the Swiss Chemical Society (SCS), which was awarded to the team of Sulzer Chemtech in March 2003.

Received: October 15, 2003

[1] M.F. Doherty, G. Buzad, Trans IchemE 1992, 70, 448.

[2] L. Götze, O. Bailer, P. Moritz, C. von Scala, Catalysis Today 2001, 69, 201. 